\title{
Strigolactones redefine plant hormones
}

\author{
Lu Wang ${ }^{1} \&$ Steven M. Smith ${ }^{1,2^{*}}$ \\ ${ }^{1}$ School of Biological Sciences, University of Tasmania, Tasmania 7001, Australia; \\ ${ }^{2}$ Institute of Genetics and Developmental Biology, Chinese Academy of sciences, Beijing 100101, China
}

Received September 3, 2016; accepted September 6, 2016; published online September 22, 2016

Citation: $\quad$ Wang, L., and Smith, S.M. (2016). Strigolactones redefine plant hormones. Sci China Life Sci 59, 1083-1085. doi: 10.1007/s11427-016-0259-5

A commonly accepted view of plant growth hormones is that their interaction with the cognate receptor protein is reversible, so that each hormone molecule can potentially interact multiple times with a receptor and so either amplify the hormone signal or maintain it over an extended period of time. The signal can be 'cancelled' by degrading the hormone. Strigolactones present us with a potentially new paradigm. The receptor is a serine hydrolase-type protein which attacks the strigolactone resulting in covalent modification of the receptor, while at the same time destroying the hormone. Strigolactone is apparently a one-use 'disposable' hormone.

Strigolactones were discovered exactly 50 years ago because they stimulate germination of parasitic weeds of the Striga genus, but they were only recognised as endogenous growth hormones in 2008 (Gomez-Roldan et al., 2008; Umehara et al., 2008). Strigolactones repress shoot branching and induce stem thickening, and they promote development of root hairs and lateral roots (Brewer et al., 2013). The effect on shoot branching provided a simple way to identify mutants impaired in strigolactone biosynthesis and perception. Many mutants were isolated in pea (Pisum sativum), rice (Oryza sativa), Arabidopsis (Arabidopsis thaliana) and petunia (Petunia hybrida) which led to the identification of the genes responsible. Two genes have been found to be required for strigolactone signaling. One of these encodes an F-box protein known as MORE AXILLARY GROWTH2 (MAX2) in Arabidopsis, DWARF3 (D3) in rice, PhMAX2 in petunia and

*Corresponding author (email: steven.smith@utas.edu.au)
RAMOSUS4 (RMS4) in pea. The other gene encodes an $\alpha / \beta$-fold hydrolase known as D14 in rice, AtD14 in Arabidopsis, DECREASED APICAL DOMINANCE2 (DAD2) in petunia and RMS3 in pea (Brewer et al., 2013; Al-Babili and Bouwmeester, 2015).

Ground-breaking research on the DAD2 protein revealed that it is able to hydrolyse the synthetic strigolactone GR24, although the rate of hydrolysis is so slow that it is essentially negligible (Hamiaux et al., 2012). More importantly, the interaction between DAD2 and GR24 led to a change in the conformation of DAD2 as revealed by differential scanning fluorimetry and by a change in its thermal stability. This change of state depended upon an intact catalytic triad (Ser, His and Asp residues) at the active site of DAD2. Furthermore, the conformational change in DAD2 led to its interaction with the F-box protein PhMAX2 in yeast two-hybrid assays (Hamiaux et al., 2012). It was proposed that this interaction led to the targeting of other proteins for ubiquitination and degradation, as happens in other hormone signalling systems such as those of jasmonates and gibberellins. Crucially, the products of GR24 hydrolysis by DAD2 are not biologically active, leading to the conclusion that the interaction between GR24 and DAD2 provides the perception mechanism and that DAD2 represents a type of 'receptor-enzyme'.

The next breakthrough came when X-ray crystallography revealed that after incubation with GR24, the rice D14 protein was covalently modified (Zhao et al., 2013). The GR24 was found to have undergone nucleophilic attack by the active site Ser of D14 and a 5-carbon moiety derived from the butenolide ring of GR24 remained covalently attached to the Ser (Zhao et al., 2013). These studies provided the 
foundation for the idea that D14 is the strigolactone receptor which becomes activated by covalent modification following attack on the strigolactone ligand. It was further reported that D14 becomes destabilised upon interaction with GR24 and D3 (Zhao et al., 2015).

Now a new study published in Nature by Yao et al. (2016) has succeeded in the major task of characterising crystals of AtD14 in association with rice D3 (the ortholog of MAX2) and the Arabidopsis ASK1 protein (to facilitate D3 solubility). The formation of crystals was induced by incubation with GR24. Analysis of the structure of the complex revealed that the active site pocket of D14 is occupied by the 5-carbon product of nucleophilic attack on the butenolide of GR24, and that the two arms of this small molecule form stable contacts with the active site Ser and His residues (Figure 1A). Subsequent digestion of D14 and isolation of peptide fragments revealed that the active site His residue was modified by covalent attachment of the 5-carbon moiety. They succeeded in isolating the same covalently modified peptide from plants, showing that it occurs in vivo. The authors propose that this 5-carbon moiety forms a stable covalent bridge between the Ser and His residues. In a parallel study on the pea RMS3 protein, strigolactone-dependent covalent modification of the His residue was also observed (de Saint Germain et al., 2016). This re- search also used a range of substrate analogs and concluded that the key to signalling is not hydrolysis but is the formation of a stable covalent intermediate. The information from these studies could prove to be vital in the design of a new generation of strigolactone mimics for use in agriculture.

The interaction of D3 protein with covalently-modified AtD14 led to a change in the conformation of both proteins (Yao et al., 2016). This could potentially facilitate the interaction of the complex with other proteins which then become targets for ubiquitination and breakdown by the 26S proteasome (Figure 1B). In a previous breakthrough by two Chinese teams, the rice D53 protein was discovered to be a target of D14 and D3 (Jiang et al., 2013; Zhou et al., 2013). D53 is a chaperone-type protein that is thought to regulate gene transcription, but the homologous proteins in Arabidopsis (SMXLs) regulate not only transcriptional responses (Wang et al., 2015), but also auxin transport (Soundappan et al., 2015) and other processes some of which might be strigolactone-independent (Liang et al., 2016). Importantly, SMXL proteins interact with D14 in a manner that depends on the D14 catalytic triad and on strigolactone (Wang et al., 2015; Liang et al., 2016). To add potential complexity or confusion, it is also reported that D14 exhibits strigolactone-dependent interaction with the

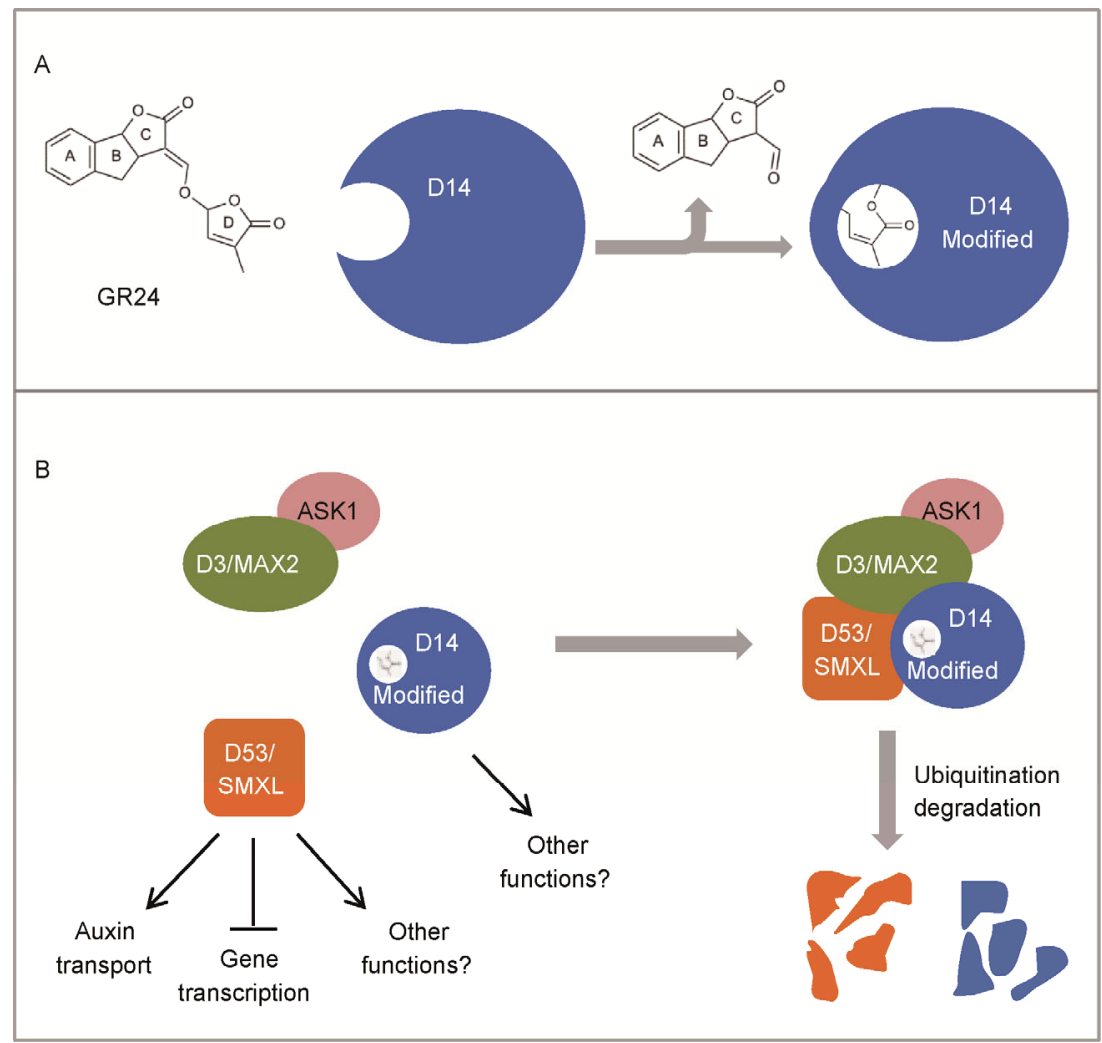

Figure 1 Covalent modification and function of strigolactone receptor D14. A. Strigolactone analog GR24 is attacked by D14, fixing the D-ring moiety in the active site pocket, which closes as a result. B. The modified D14 interacts with D3/MAX2 and D53/SMXL to form a ubiquitination complex. Note that D14 undergoes a conformational change when complexed with D3/MAX2. This leads to the degradation of both D53/SMXL and D14. The destruction of D53/SMXL leads to changes in cell function including auxin transport and gene expression. D14 might have other functions, such as interaction with DELLA proteins (not shown). 
rice DELLA protein SLR1, which could therefore provide a mechanism for crosstalk with gibberellin signalling (Nakamura et al., 2013).

Since D14 interactions with D3/MAX2 were discovered before D14 interactions with D53/SMXLs, attention has been focused mainly on the D14-D3/MAX2 interaction as the primary event in strigolactone signalling. However we now need to consider the possibility that D14 could interact with D53/SMXL proteins before interacting with D3/MAX2, or even interact independently. The D14 and D3/MAX2 proteins are observed in both cytosol and nucleus, while D53/SMXL proteins are nuclear (Liang et al., 2016). One scenario is that D14 interacts first with strigolactone in the cytosol to create the covalently modified D14. Intriguingly this modification to D14 might provide a signal for its translocation to the nucleus, potentially in a complex with D3/MAX2. Another question is whether D14 and D3/MAX2 assemble with D53/SMXL while it is bound to the chromosome, since ubiquitination complexes are known to operate on chromatin proteins in situ. It is also known that D14 is degraded in a MAX2-dependent process following its interaction with strigolactone (Chevalier et al., 2014). This raises the possibility that not only is strigolactone destroyed during the signalling process, but its receptor protein might suffer the same fate. The discoveries of Yao et al. (2016) will open up ways to address such exciting questions. Furthermore, while GR24 is a very active strigolactone analog, the natural endogenous strigolactone signal is unknown, but is likely to be a derivative of carlactonoic acid rather than a canonical strigolactone of the type that is secreted from roots (Al-Babili and Bouwmeester, 2015). This raises another intriguing question about the fate of the non-butenolide part of the endogenous hormone, and whether it might have a separate signalling function.

The rapid progress in understanding the function of strigolactones has been a great success story in recent scientific research. The revelation that strigolactones operate by means of a novel mechanism that covalently modifies the receptor and simultaneously destroys the hormone makes us re-think our understanding of plant hormones. Do hormone molecules typically trigger multiple signalling events before they are broken down, or are they more like the 'disposable' strigolactones?

Compliance and ethics The author(s) declare that they have no conflict of interest.

Al-Babili, S., and Bouwmeester, H.J. (2015). Strigolactones, a novel carotenoid-derived plant hormone. Annu Rev Plant Biol 66, 161-186.

Brewer, P.B., Koltai, H., and Beveridge, C.A. (2013). Diverse roles of strigolactones in plant development. Mol Plant 6, 18-28.

Chevalier, F., Nieminen, K., Sánchez-Ferrero, J.C., Rodríguez, M.L., Chagoyen, M., Hardtke, C.S., and Cubas, P. (2014). Strigolactone pro- motes degradation of DWARF14, an $\alpha / \beta$ hydrolase essential for strigolactone signaling in Arabidopsis. Plant Cell 26, 1134-1150.

Gomez-Roldan, V., Fermas, S., Brewer, P.B., Puech-Pagès, V., Dun, E.A., Pillot, J.P., Letisse, F., Matusova, R., Danoun, S., Portais, J.C., Bouwmeester, H., Bécard, G., Beveridge, C.A., Rameau, C., and Rochange, S.F. (2008). Strigolactone inhibition of shoot branching. Nature $11,189-194$.

Hamiaux, C., Drummond, R.S., Janssen, B.J., Ledger, S.E., Cooney, J.M., Newcomb, R.D. and Snowden, K.C. (2012). DAD2 is an $\alpha / \beta$ hydrolase likely to be involved in the perception of the plant branching hormone, strigolactone. Curr Biol 6, 2032-2036.

Jiang, L., Liu, X., Xiong, G., Liu, H., Chen, F., Wang, L., Meng, X., Liu, G., Yu, H., Yuan, Y., Yi, W., Zhao, L., Ma, H., He, Y., Wu, Z., Melcher, K., Qian, Q., Xu, H.E., Wang, Y., and Li, J. (2013). DWAFR53 acts as a repressor of strigolactone signalling in rice. Nature 504, 410-415.

Liang, Y., Ward, S., Li, P., Bennett, T., and Leyser, O. (2016). SMAX1-LIKE7 signals from the nucleus to regulate shoot development in Arabidopsis via partially EAR motif-independent mechanisms. Plant Cell 28, 1581-1601.

Nakamura, H., Xue, Y.L., Miyakawa, T., Hou, F., Qin, H.M., Fukui, K., Shi, X., Ito, E., Ito, S., Park, S.H., Miyauchi, Y., Asano, A., Totsuka, N., Ueda, T., Tanokura, M., and Asami, T. (2013). Molecular mechanism of strigolactone perception by DWARF14. Nat Commun 4, 2613.

de Saint Germain, A., Clavé, G., Badet-Denisot, M.A., Pillot, J.P., Cornu, D., Le Caer, J.P., Burger, M., Pelissier, F., Retailleau, P., Turnbull, C., Bonhomme, S., Chory, J., Rameau, C., and Boyer, F.D. (2016). An histidine covalent receptor and butenolide complex mediates strigolactone perception. Nat Chem Biol, doi: 10.1038/nchembio.2147.

Soundappan, I., Bennett, T., Morffy, N., Liang, Y., Stanga, J.P., Abbas, A., Leyser, O., and Nelson, D.C. (2015). SMAX1-LIKE/D53 family members enable Distinct MAX2-dependent responses to strigolactones and karrikins in Arabidopsis. Plant Cell 27, 3143-3159.

Umehara, M., Hanada, A., Yoshida, S., Akiyama, K., Arite, T., Takeda-Kamiya, N., Magome, H., Kamiya, Y., Shirasu, K., Yoneyama, K., Kyozuka, J., and Yamaguchi, S. (2008). Inhibition of shoot branching by new terpenoid plant hormones. Nature 11, 195-200.

Wang, L., Wang, B., Jiang, L., Liu, X., Li, X., Lu, Z., Meng, X., Wang, Y., Smith, S.M., and Li, J. (2015). Strigolactone signaling in Arabidopsis regulates shoot development by targeting D53-Like SMXL repressor proteins for ubiquitination and degradation. Plant Cell 27, 3128-3142.

Yao, R., Ming, Z., Yan, L., Li, S., Wang, F., Ma, S., Yu, C., Yang, M., Chen, L., Chen, L., Lu, Y., Yan, C., Miao, D., Sun, Z., Yan, J., Sun, Y., Wang, L., Chu, J., Fan, S., He, W., Deng, H., Nan, F., Li, J., Rao, Z., Lou, Z., and Xie, D. (2016). DWARF14 is a non-canonical hormone receptor for strigolactone. Nature 536, 469-473.

Zhao, L.H., Zhou, X.E., Wu, Z.S., Yi, W., Xu, Y., Li, S., Xu, T.H., Liu, Y., Chen, R.Z., Kovach, A., Kang, Y., Hou, L., He, Y., Xie, C., Song, W., Zhong, D., Xu, Y., Wang, Y., Li, J., Zhang, C., Melcher, K., and Xu, H.E. (2013). Crystal structures of two phytohormone signal-transducing $\alpha / \beta$ hydrolases: karrikin-signaling KAI 2 and strigolactone-signaling DWARF14. Cell Res 23, 436-439.

Zhao, L.H., Zhou, X.E., Yi, W., Wu, Z., Liu, Y., Kang, Y., Hou, L., de Waal, P.W., Li, S., Jiang, Y., Scaffidi, A., Flematti, G.R., Smith, S.M., Lam, V.Q., Griffin, P.R., Wang, Y., Li, J., Melcher, K., and Xu, H.E. (2015). Destabilization of strigolactone receptor DWARF14 by binding of ligand and E3-ligase signalling effector DWARF3. Cell Res 25, 1219-1236.

Zhou, F., Lin, Q., Zhu, L., Ren, Y., Zhou, K., Shabek, N., Wu, F., Mao, H., Dong, W., Gan, L., Ma, W., Gao, H., Chen, J., Yang, C., Wang, D., Tan, J., Zhang, X., Guo, X., Wang, J., Jiang, L., Liu, X., Chen, W., Chu, J., Yan, C., Ueno, K., Ito, S., Asami, T., Cheng, Z., Wang, J., Lei, C., Zhai, H., Wu, C., Wang, H., Zheng, N., and Wan, J. (2013). D14-SCF(D3)-dependent degradation of D53 regulates strigolactone signalling. Nature 504, 406-410.

Open Access This article is distributed under the terms of the Creative Commons Attribution License which permits any use, distribution, and reproduction in any medium, provided the original author(s) and source are credited. 